\title{
Asthma-COPD overlap syndrome in the US: a prospective population-based analysis of patient- reported outcomes and health care utilization
}

This article was published in the following Dove Press journal:

International Journal of COPD

3 February 2017

Number of times this article has been viewed

\author{
Carlos A Vaz Fragoso',2 \\ Terrence E Murphy' \\ George O Agogo' \\ Heather G Allore ${ }^{1,3}$ \\ Gail J McAvay'
}

'Department of Medicine, Yale School of Medicine, New Haven, ${ }^{2}$ Veterans Affairs Clinical Epidemiology Research Center, West Haven, ${ }^{3}$ Department of Biostatistics, Yale School of Public Health, New Haven, CT, USA
Correspondence: Carlos A Vaz Fragoso Veterans Affairs Clinical Epidemiology Research Center, 950 Campbell Avenue, West Haven, CT 06516, USA

Tel +l 2036889423

Fax +l 2036884209

Email carlos.fragoso@yale.edu
Background: Prior work suggests that asthma-COPD overlap syndrome (ACOS) has a greater health burden than asthma alone or COPD alone. In the current study, we have further evaluated the health burden of ACOS in a nationally representative sample of the US population, focusing on patient-reported outcomes and health care utilization and on comparisons with asthma alone and COPD alone. Patient-reported outcomes are especially meaningful, as these include functional activities that are highly valued by patients and are the basis for patient-centered care.

Methods: Using data from the Medical Expenditure Panel Survey (MEPS), we evaluated patientreported outcomes and health care utilization among participants who were aged 40-85 years and had self-reported, physician-diagnosed asthma or COPD. MEPS administered five rounds of interviews, at baseline and approximately every 6 months over 2.5 years. Patient-reported outcomes included activities of daily living (ADLs), mobility, social/recreational activities, disability days in bed, and health status (Short Form 12, Version 2). Health care utilization included outpatient and emergency department (ED) visits, and hospitalization.

Results: Of 3,486 participants with asthma or COPD, 1,585 (45.4\%) had asthma alone, 1,294 (37.1\%) had COPD alone, and 607 (17.4\%) had ACOS. Relative to asthma alone, ACOS was significantly associated with higher odds of prevalent disability in ADLs and limitations in mobility and social/recreational activities (adjusted odds ratios [adjORs]: 1.91-3.98), as well as with higher odds of incident limitations in mobility and social/recreational activities, disability days in bed, and respiratory-based outpatient and ED visits, and hospitalization (adjORs: 1.86-2.35). In addition, ACOS had significantly worse physical and mental health scores than asthma alone ( $P$-values $<0.0001)$. Relative to COPD alone, ACOS was significantly associated with higher odds of prevalent limitations in mobility and social/recreational activities (adjORs: 1.68-2.06), as well as with higher odds of incident disability days in bed and respiratory-based outpatient and ED visits (adjORs: 1.48-1.74). In addition, ACOS had a significantly worse physical health score, but similar mental health score, as compared with COPD alone ( $P$-values 0.0025 and 0.1578 , respectively).

Conclusion: In the US, ACOS is associated with a greater health burden, including patientreported outcomes and health care utilization, relative to asthma alone and COPD alone.

Keywords: disability, mobility, activities of daily living, social, recreational

\section{Introduction}

In the US, obstructive airway disease in middle-aged and older persons occurs most often as asthma or COPD. ${ }^{1-5}$ During the period from 2008 through 2010, the US National Surveillance of Asthma reported the prevalence of current asthma as $7.7 \%$ in 
those aged 35-64 years, as well as in those aged $\geq 65$ years. ${ }^{4}$ Current asthma was defined epidemiologically as a selfreported physician diagnosis still present at the time of the survey, and in the stated age groups, women were more likely to self-report asthma. ${ }^{4}$ In the year 2011, the US Behavioral Risk Factor Surveillance System reported a prevalence of COPD that ranged from $6.6 \%$ to $9.2 \%$ across the age group of 45-64 years and from $11.6 \%$ to $12.1 \%$ across the age group of $\geq 65$ years. ${ }^{5}$ COPD was defined epidemiologically as a self-reported physician diagnosis of chronic bronchitis or emphysema, and in the stated age groups, women were more likely to self-report COPD. ${ }^{5}$ In general, asthma is characterized by variable, reversible airway obstruction, frequently associated with an atopic history, while COPD is characterized by progressive, irreversible airway obstruction, frequently due to tobacco smoking. ${ }^{1-3}$

Among middle-aged and older adults with asthma or COPD, $15 \%-45 \%$ have both conditions, referred to as the asthma-COPD overlap syndrome (ACOS). ${ }^{1,6-12}$ In particular, ACOS may include long-standing or adult-onset asthma that has progressed to irreversible airway obstruction (a consequence of airway remodeling from chronic airway inflammation that is specific to allergens, but can also be nonspecific), as well as include COPD that is characterized by having both a smoking and atopic history, and a reversible component to the airway obstruction. ${ }^{1-3,6-17}$

Prior work has suggested that ACOS is associated with a greater health burden, 1,6-12,15-25 including respiratory symptoms, health-related quality of life, exacerbations, and comorbidities, as compared with asthma alone and COPD alone. In the current study, we have further evaluated the health burden of ACOS in a nationally representative sample of the US population, focusing on patient-reported outcomes and health care utilization, and on comparisons with asthma alone and COPD alone. Patient-reported outcomes are especially meaningful, as these often include functional activities that are highly valued by patients and are the basis for patient-centered care. ${ }^{26-28}$

To evaluate the health burden in ACOS, we have used baseline and longitudinal data from the Medical Expenditure Panel Survey (MEPS), a large, nationally representative sample of the US population. ${ }^{29}$ MEPS included epidemiologic definitions of asthma and COPD, as described earlier, and a broad array of demographic and clinical characteristics. Patient-reported outcomes were also evaluated, having included disability in self-care activities of daily living (ADLs), limitations in mobility and social/recreational activities, disability days in bed, and health status. Health care utilization was additionally evaluated in several clinical settings, having included outpatient and emergency department (ED) visits, and hospitalizations (identified by diagnostic codes from the International Classification of Diseases, Ninth Revision [ICD-9]).

\section{Methods Study population}

MEPS cohort is a nationally representative sample of the US civilian population, sponsored by the US Department of Health and Human Services (DHHS) through the Agency for Healthcare Research and Quality (AHRQ) and the Centers for Disease Control and Prevention (CDC). Participants were followed for 2.5 years, with five rounds of interviews occurring approximately every 6 months after the baseline visit (round 1). The data for the current study came from the Household Component of the survey, administered at baseline, wherein one adult from the household is questioned about demographic and clinical characteristics of household members, respiratory medications, functional status, and health care utilization (outpatient and ED visits, and hospitalizations). A self-administered questionnaire was additionally completed by each member of the household at round 2 of the survey to assess smoking history, sensory impairments (vision and hearing), and depressive symptoms.

In the current study, the analytical sample included participants from the 2008-2012 panels of MEPS who were aged 40-85 years, had self-reported, physician-diagnosed COPD or asthma, and who gave information on smoking history (Figure 1). ${ }^{29}$ We selected age $\geq 40$ years because COPD and its related adverse health outcomes are most prevalent in this age group. ${ }^{5,30}$ Because the study used existing de-identified data that were publicly available, it was granted exemption from participant consent and ethical approval by the institutional review board of Yale University.

\section{Asthma, COPD, and ACOS}

The diagnoses of asthma and COPD were established at the baseline visit, based on data from the priority condition section of the household interview. Asthma was defined as a self-reported physician diagnosis that was still present at the baseline interview (current asthma). COPD was defined as self-reported chronic bronchitis (present in the 12 months prior to the baseline interview) or emphysema (ever-diagnosed). ACOS was then defined as having both asthma and COPD.

\section{Demographic and clinical characteristics}

Demographic characteristics included age (40-64 vs 65-85 years), sex, race (black vs other), ethnicity (Hispanic 


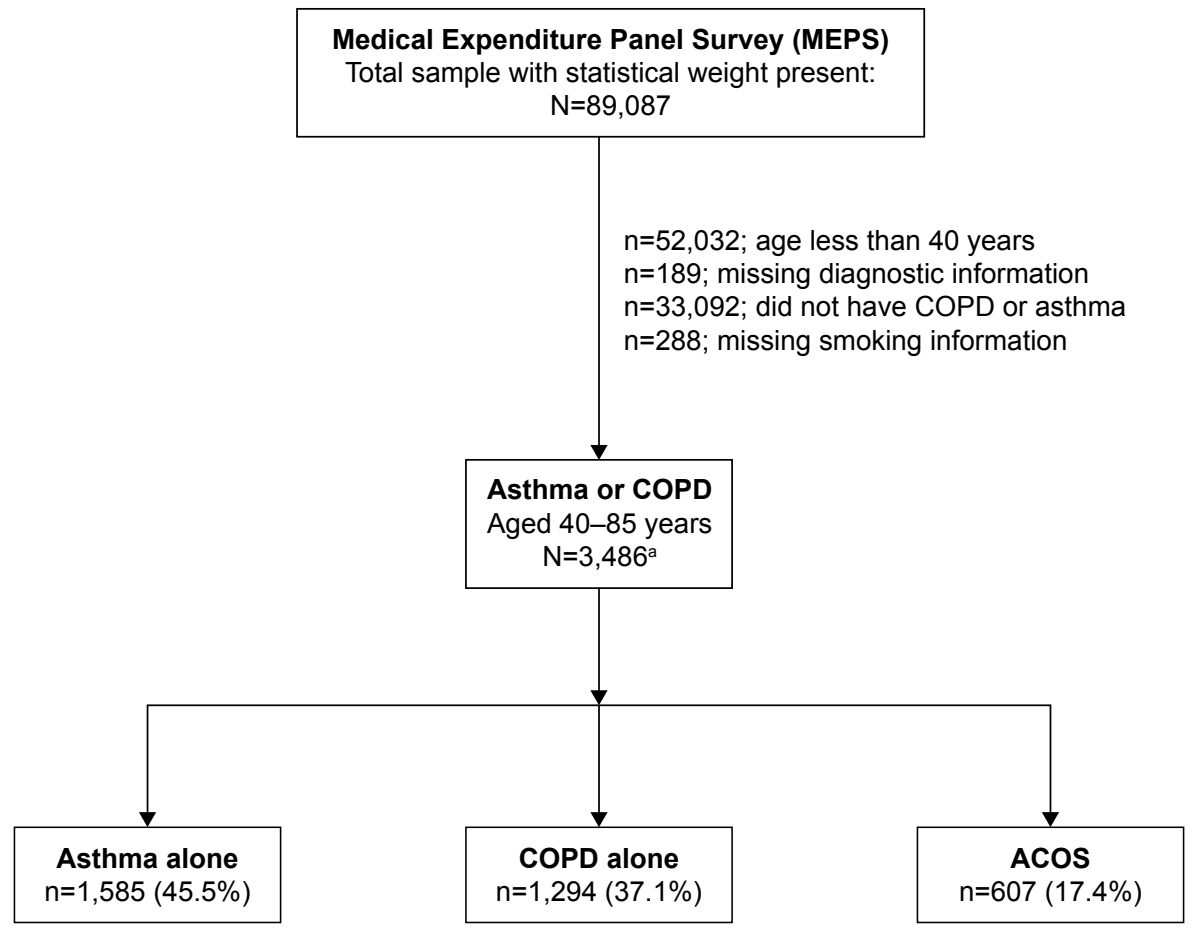

Figure I Sample size of the MEPS: 2008-2012 panels.

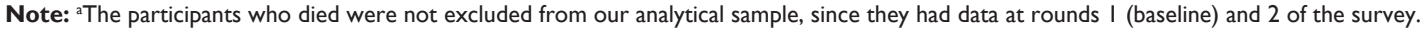
Abbreviations: MEPS, Medical Expenditure Panel Survey; ACOS, asthma-COPD overlap syndrome.

vs other), marital status (married vs not married), education ( $\leq 11$ vs $\geq 12$ years), income (poor-to-low vs middle-to-high income, defined by $<200 \%$ vs $\geq 200 \%$ of family income divided by the poverty line, respectively), having private medical insurance during the first study year, and smoking status (current vs never or former smoker). Comorbid conditions included self-reported, physician-diagnosed cardiovascular disease (coronary heart disease, angina, or myocardial infarction), stroke, diabetes, and cancer (any). Depressive symptomatology was defined by a Patient Health Questionnaire score $\geq 3 .{ }^{31}$ Impairments were also assessed in cognition (confusion, memory loss, difficulty making decisions, or requiring supervision for safety), vision (ranging from some difficulty to not being able to read a newspaper or recognize familiar people standing 2-3 feet away), and hearing (ranging from some difficulty to cannot hear most things people say). Vision and hearing impairments were combined into sensory impairment, if either or both were present. Of note, smoking history based on pack-years and respiratory symptoms, such as dyspnea and wheezing, were unavailable in MEPS.

As described earlier, demographic and clinical characteristics were evaluated at baseline (round 1), but current smoking status, depressive symptomatology, and sensory impairments were first evaluated at round 2. However, if a current smoker was identified at round 2 , that person was assumed to have been a smoker at baseline (round 1), given the adult age of participants (smoking is typically initiated prior to middle age) and the close temporal proximity of rounds 1 and 2 .

\section{Respiratory medications}

Respiratory medications were identified at the baseline visit by bottles or receipts and categorized according to therapeutic class and subclass using the Multum Lexicon system from Cerner Multum, Inc (Denver, CO, USA; www.multum.

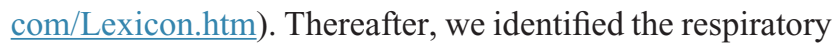
medications based on these codes, confirming all medication names for accuracy.

\section{Patient-reported outcomes}

The outcomes of interest included disability in self-care ADLs (evaluated at baseline and over 2.5 years of follow-up), limitations in mobility and social/recreational activities (evaluated at baseline and over 2.5 years of follow-up), number of disability days in bed that were due to physical or mental problems (evaluated over 2.5 years of follow-up), and health status (evaluated at rounds 2 and 4). ADL disability was defined as needing help or supervision with bathing, dressing, or getting around the house. Mobility limitations were defined as difficulty in walking up 10 steps and in walking 
three blocks. A social/recreation limitation was established if participation in social, recreational, or family activities was limited as a result of impairments or a physical or mental health problem. The number of disability days spent in bed was ascertained over a follow-up period of 2.5 years, stratified as 0-6 days and $\geq 7$ days. Health status was evaluated by the Short Form 12, Version 2 (SF-12v2), which included 12 items from the Medical Outcomes Study, 36-Item ShortForm Health Survey and which yielded scores for a Physical Component Summary (PCS) and a Mental Component Summary (MCS). ${ }^{32}$ The PCS score measured the participant's perceived impact of physical health on activities, while the MCS score measured the participant's perceived impact of mental health on activities. ${ }^{32}$ In normative data, the mean score is set to 50; thus, PCS and MCS scores $<50$ indicate worse physical and mental health, respectively. ${ }^{32}$

\section{Health care utilization}

Outpatient visits (including physician or hospital clinic visits), ED visits, and hospitalizations were additionally ascertained over 2.5 years of follow-up, with each categorized by ICD-9 codes as cardiac $(401,410,413-415,423-428,431,433-438$, 440-444, and 447), respiratory $(416,464,466,471-473$, 477, 478, 480, 482, 483, 485-488, 490-493, 496, 505, 511, 514,515 , and 518), and for any reason, respectively. The outpatient visits were further stratified based on a threshold of having six or more outpatient visits, corresponding to a frequency averaging more often than every 6 months over 2.5 years of follow-up.

\section{Statistical analysis}

Unless stated otherwise, comparisons were made between ACOS and asthma alone and between ACOS and COPD alone, with adjustments made for primary sampling units, strata, and weights of the MEPS data; these sample weights provide the US population-level estimates.

First, the demographic and clinical characteristics of asthma, COPD, and ACOS were evaluated at baseline in a single multinomial logistic multivariable model that was adjusted for the following covariates: age, gender, black race, Hispanic ethnicity, marital status, education, income, medical insurance, current smoking status, comorbid conditions, and cognitive and sensory impairment. Correlation among covariates was all $<0.3$ with Kendall's Tau b. Next, the use of respiratory medications at baseline was evaluated using unadjusted logistic regression models; $P$-values were adjusted for multiple comparisons (Hochberg method). The prevalence of patient-reported outcomes at baseline was also evaluated using multivariable logistic regression models that were adjusted for the baseline covariates described earlier.

Among participants who were non-disabled or were without limitations at baseline, the onset of patient-reported outcomes, measured approximately every 6 months over a period of 2.5 years, was additionally evaluated. Specifically, the respective associations with incident disability in ADLs, incident limitations in mobility and social/recreational activities, and incident disability days in bed ( $\geq 7$ vs 0-6 days) - over the follow-up period of 2.5 years - were tested with multivariable logistic regression models, adjusted for the same baseline covariates as described earlier. For all participants, three classes of health care utilization (cardiac, respiratory, and any) were evaluated using multivariable logistic regression models, adjusted for the same baseline covariates as described earlier. Finally, for all participants, the PCS and MCS scores from the SF-12v2 were evaluated separately, using longitudinal linear modeling adjusted for time and the baseline covariates described earlier, and the results are presented as adjusted least squares mean scores with $95 \%$ confidence intervals.

Missing baseline data were limited to four characteristics (education, comorbid cardiovascular disease, cognition, and sensory impairment), all $<0.01 \%$. The amount of missingness for all outcomes except disability days was less than $1 \%$, while disability days was missing for less than $2 \%$ of the sample. Because of this small amount of missing data, all modeling of outcomes was based on complete case analysis.

All statistical analyses were performed using SAS software version 9.4 (SAS Institute, Cary, NC, USA), with a two-sided $\alpha=0.05$ for statistical significance.

\section{Results}

Figure 1 shows the sample size of MEPS for the 2008-2012 panels. Of the 3,486 participants who had asthma or COPD, 1,585 (45.4\%) had asthma alone, 1,294 (37.1\%) had COPD alone, and 607 (17.4\%) had ACOS (included both asthma and COPD). Over the follow-up period of 2.5 years, $98(2.8 \%)$ participants had died.

Table 1 shows the demographic and clinical characteristics, with percentages representing the MEPS national frequencies. Relative to asthma alone, ACOS had significantly higher frequencies of older age, less education, current smoker status, cardiovascular disease, cancer, and depressive symptomatology, but a lower frequency of Hispanic representation. Relative to COPD alone, ACOS had significantly higher frequencies of female representation 
Table I Baseline demographic and clinical characteristics: asthma alone, COPD alone, and ACOS

\begin{tabular}{|c|c|c|c|c|c|}
\hline \multirow[t]{2}{*}{ Characteristics $^{\mathrm{a}}$} & \multicolumn{3}{|c|}{ Percent prevalence (\%) } & \multicolumn{2}{|l|}{$P$-value ${ }^{c}$} \\
\hline & Asthma $(\mathrm{N}=I, 585)$ & $\operatorname{COPD}(\mathrm{N}=1,294)$ & $\operatorname{Acos}(\mathrm{N}=607)$ & ACOS vs asthma & ACOS vs COPD \\
\hline \multicolumn{6}{|l|}{ Age (years) } \\
\hline 40-64 (middle age) & 77.0 & 50.7 & 63.2 & $<0.001$ & $<0.00 \mathrm{I}$ \\
\hline 65-85 (older age) & 23.0 & 49.3 & 36.7 & & \\
\hline Female & 70.6 & 51.7 & 67.8 & 0.692 & $<0.00 \mathrm{I}$ \\
\hline Black & 12.6 & 7.6 & 11.0 & 0.070 & 0.288 \\
\hline Hispanic & 8.7 & 6.0 & 6.6 & 0.019 & 0.650 \\
\hline Married & 57.9 & 54.3 & 43.8 & 0.072 & 0.044 \\
\hline Education: $<$ high school & 12.5 & 26.6 & 29.7 & $<0.001$ & 0.455 \\
\hline Low income & 30.7 & 39.6 & 45.7 & 0.969 & 0.947 \\
\hline Medical insurance & 90.8 & 93.7 & 90.2 & 0.620 & 0.165 \\
\hline Current smoker & 14.0 & 39.3 & 38.1 & $<0.001$ & 0.038 \\
\hline \multicolumn{6}{|l|}{ Comorbid conditions } \\
\hline Cardiovascular $^{\mathrm{d}}$ & 13.1 & 29.7 & 34.7 & $<0.001$ & 0.050 \\
\hline Stroke & 7.8 & 11.9 & 15.4 & 0.784 & 0.307 \\
\hline Diabetes & 18.2 & 20.9 & 26.5 & 0.288 & 0.142 \\
\hline Cancer & 16.7 & 27.0 & 25.6 & 0.013 & 0.933 \\
\hline Depressive symptomatologye & 13.0 & 23.0 & 30.4 & $<0.001$ & 0.045 \\
\hline \multicolumn{6}{|l|}{ Impairments } \\
\hline Cognitive & 9.6 & 16.7 & 19.5 & 0.733 & 0.501 \\
\hline Sensory ${ }^{f}$ & 19.2 & 32.8 & 29.6 & 0.115 & 0.367 \\
\hline
\end{tabular}

Notes: aEvaluated at the baseline visit (round I of the survey), except for smoking history, depressive symptomatology, and sensory impairments (first evaluated in round 2). 'Percentages are adjusted for sampling weights but unadjusted for other factors in the model. 'From a multinomial logistic regression model adjusted for all characteristics in the table. Includes coronary heart disease, myocardial infarction, and angina. ePatient Health Questionnaire score $\geq 3$. Includes vision or hearing.

Abbreviation: ACOS, asthma-COPD overlap syndrome.

and depressive symptomatology, but lower frequencies of older age and married status. Otherwise, frequencies of cardiovascular disease and current smoker status in ACOS vs COPD alone were only borderline significant or minimally different (respectively).

Table 2 shows the respiratory medications, with percentages representing the MEPS national frequencies. Relative to asthma alone, ACOS had significantly higher frequencies of using a steroid (systemic and inhaled) and bronchodilator (all three classes). Relative to COPD alone, ACOS had significantly higher frequencies of using a steroid (systemic and inhaled), bronchodilator (adrenergic or anticholinergic), leukotriene modifier, antihistamine, and decongestant.

Figure 2 shows the adjusted odds ratios for patientreported outcomes, at baseline (prevalent) and over a follow-up period of 2.5 years (incident). Relative to asthma alone, ACOS was significantly associated with higher odds of having prevalent disability in self-care ADLs and limitations in mobility and social/recreational activities (adjusted odds ratios: 1.91-3.98), as well as higher odds of having incident limitations in mobility and social/recreational activities, and disability days in bed (adjusted odds ratios: 1.86-2.35). Relative to COPD alone, ACOS was significantly associated with higher odds of having prevalent limitations in mobility and social/recreational activities (adjusted odds ratios: 1.68-2.06), as well as higher odds of having incident disability days in bed (adjusted odds ratio: 1.48).

Figure 3 shows the adjusted odds ratios for health care utilization, over a follow-up period of 2.5 years. Relative to asthma alone, ACOS was significantly associated with higher odds of having respiratory-based outpatient and ED visits, and respiratory-based hospitalization (adjusted odds ratios: 2.38-2.86), as well as higher odds of having any outpatient and ED visits, and any hospitalization (adjusted odds ratios: 1.53-1.75). Relative to COPD alone, ACOS was significantly associated with higher odds of having respiratory-based outpatient and ED visits (adjusted odds ratios: 1.74 and 1.64, respectively). Otherwise, cardiac-based health care utilization only differed for outpatient visits and only between ACOS and asthma alone (adjusted odds ratio: 1.45).

Table 3 shows the health status, based on adjusted least squares mean scores for the PCS and MCS of the SF-12v2. The adjusted least squares mean scores for the PCS and MCS were less than 50 (signifying worse health status) in asthma alone, COPD alone, and ACOS. Relative to asthma alone, ACOS had significantly lower adjusted least squares mean scores for the PCS and MCS ( $P$-values $<0.0001)$. Relative to COPD alone, ACOS had significantly lower adjusted 
Table 2 Respiratory medication use: asthma alone, COPD alone, and ACOS

\begin{tabular}{|c|c|c|c|c|c|}
\hline \multirow[t]{2}{*}{ Respiratory medications } & \multicolumn{3}{|c|}{ Percent prevalence $(\%)^{\mathrm{a}}$} & \multicolumn{2}{|l|}{$P$-value ${ }^{b}$} \\
\hline & Asthma $(\mathrm{N}=\mathrm{I}, 585)$ & COPD $(\mathrm{N}=\mathrm{I}, 294)$ & $A \cos (N=607)$ & ACOS vs asthma & ACOS vs COPD \\
\hline \multicolumn{6}{|l|}{ Steroids } \\
\hline Systemic & 4.1 & 5.9 & II.I & $<0.001$ & 0.007 \\
\hline Inhaled & 19.1 & 10.7 & 26.6 & 0.042 & $<0.001$ \\
\hline \multicolumn{6}{|l|}{ Bronchodilators $^{c}$} \\
\hline Adrenergic $^{d}$ & 26.3 & 18.0 & 41.7 & $<0.001$ & $<0.001$ \\
\hline Anticholinergic $^{e}$ & 3.0 & 10.7 & 16.0 & $<0.001$ & 0.035 \\
\hline Methylxanthine ${ }^{f}$ & 0.5 & 0.8 & 2.5 & 0.018 & 0.056 \\
\hline Any & 27.2 & 20.8 & 44.4 & $<0.001$ & $<0.001$ \\
\hline \multicolumn{6}{|l|}{ Smoking cessation } \\
\hline Nicotine supplement & 0.03 & 0.2 & 0.1 & NA & \\
\hline Nicotine receptor agonist & 0.2 & 0.7 & 0.8 & & \\
\hline \multicolumn{6}{|l|}{ Asthma-based medication } \\
\hline Cromolyn oral inhaler & 0.01 & 0 & 0 & NA & \\
\hline Leukotriene modifier & 9.6 & 2.7 & 10.6 & 0.552 & $<0.001$ \\
\hline Any & 9.6 & 2.7 & 10.6 & 0.552 & $<0.001$ \\
\hline Antihistamine & 9.5 & 5.8 & 12.7 & 0.524 & $<0.001$ \\
\hline Decongestant & 1.2 & 0.7 & 2.3 & 0.524 & 0.004 \\
\hline Expectorant & 1.8 & 1.4 & 2.3 & 0.552 & 0.426 \\
\hline Cough suppressant & 2.1 & 2.4 & 3.0 & 0.552 & 0.503 \\
\hline \multicolumn{6}{|l|}{ Nasal sprays } \\
\hline Steroid nasal spray & 6.1 & 3.4 & 7.4 & 0.552 & 0.056 \\
\hline Cromolyn nasal spray & 0 & 0 & 0 & NA & \\
\hline
\end{tabular}

Notes: Some data were NA due to small cell size. aPercentages are adjusted for sampling weights but unadjusted for other factors in the model. ${ }^{\mathrm{b}} \mathrm{Comparisons} \mathrm{were} \mathrm{ACOS}$ vs asthma alone and ACOS vs COPD alone, using unadjusted logistic regression models; $P$-values were, however, adjusted for multiple comparisons by the Hochberg method (these adjustments may result in identical $P$-values). 'Stratified by pharmacologic category. ${ }^{\mathrm{d} B e t a}{ }_{2}$-selective, aerosolized (inhaler or nebulizer), or oral formulation. eAerosolized (inhaler or nebulizer). 'In addition to bronchodilation, methylxanthines may have other putative effects (eg, increased diaphragmatic muscle strength); available in multiple oral formulations.

Abbreviations: ACOS, asthma-COPD overlap syndrome; NA, not applicable.

least squares mean score for the PCS $(P$-value 0.0025$)$ but a similar adjusted least squares mean score for the MCS ( $P$-value 0.1578$)$.

\section{Discussion}

In a large, nationally representative sample of the US population aged 40-85 years (MEPS), our results reaffirm that ACOS is associated with increased health burden, relative to asthma alone and COPD alone. In particular, ACOS was consistently associated with patient-reported outcomes, characterized by increased limitations in mobility and social/ recreational activities, increased disability days in bed, and worse physical health status. Moreover, ACOS was consistently associated with increased respiratory-based outpatient and ED visits. In these associations, prevalent and incident health burden were considered similarly important, because irreversible obstructive airway disease in middle-aged or older persons may have already resulted in persistent adverse health effects at the baseline visit.

The increased health burden in ACOS, relative to asthma alone and COPD alone, may be due to an underlying respiratory mechanism. Specifically, our results show that the health burden in ACOS was additionally characterized by significantly higher frequencies of using a steroid (systemic and inhaled) and bronchodilator at the baseline visit, as compared with asthma alone and COPD alone. Increased use of respiratory medications in ACOS, together with increased respiratory-based outpatient and ED visits, strongly suggests that a respiratory mechanism contributed to the increased health burden in ACOS, relative to asthma alone and COPD alone.

The respiratory mechanism that underlies the increased health burden in ACOS may have been due to obstructive airway disease. ${ }^{1-3,6-17} \mathrm{We}$ found, for example, that the use of allergy-related medications (leukotriene modifier and antihistamine) was significantly more frequent in ACOS than COPD alone, and that older age and current smoker status were significantly more frequent in ACOS than asthma alone. Hence, we postulate that having an atopic and smoking history, as well as being middle-aged or older, may have driven the pathophysiology of ACOS to include obstructive airway disease that is due to coexisting COPD and 
A

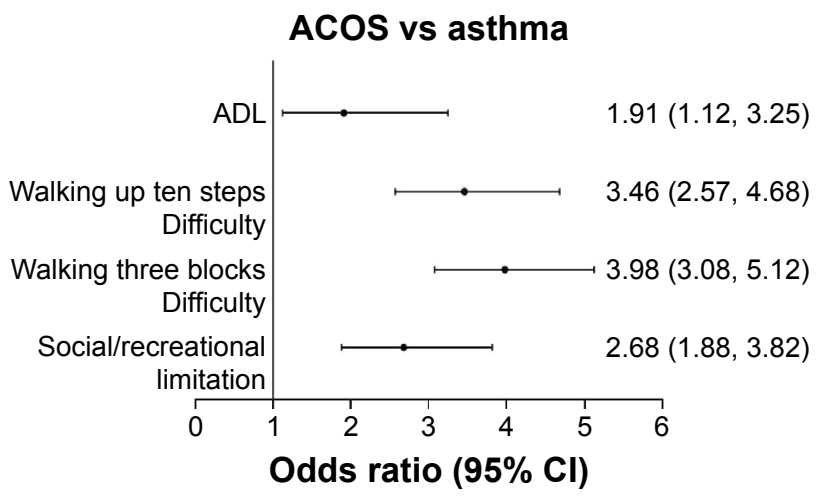

\section{Prevalent $^{\mathrm{a}}$}

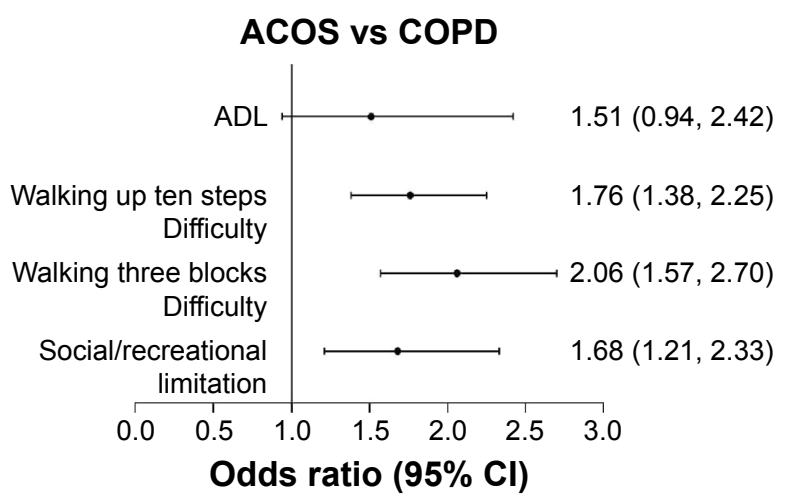

B

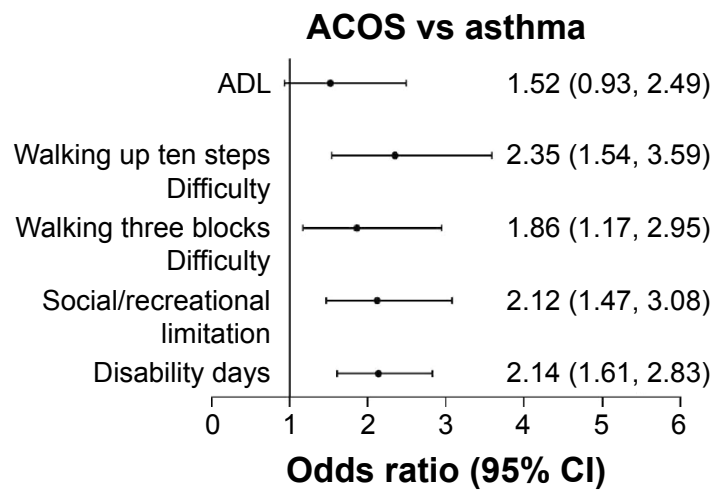

Incident ${ }^{\mathbf{b}}$

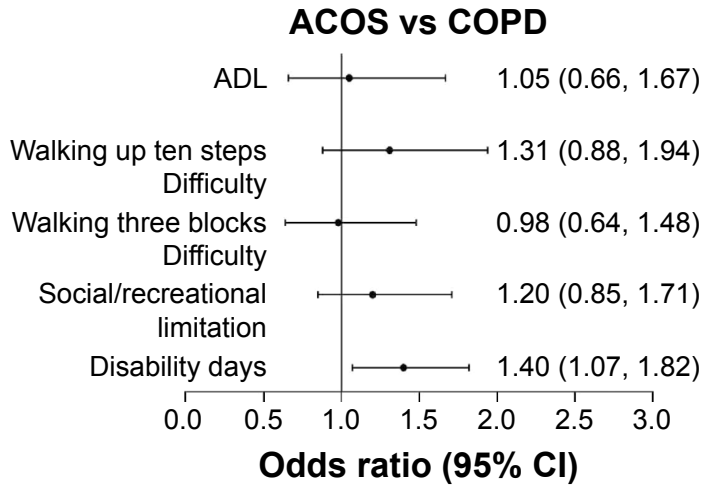

Figure 2 Comparisons of patient-reported outcomes at baseline and over a follow-up period of 2.5 years: ACOS vs asthma alone and ACOS vs COPD alone. Notes: aEvaluated at baseline and included disability in self-care ADLs and limitations in mobility and social/recreational activities. Comparisons were made between ACOS vs asthma alone and ACOS vs COPD alone, using multivariable logistic regression models that were adjusted for baseline covariates (age, sex, race, ethnicity, marital status, education, income, medical insurance status, current smoking status, cardiovascular diseases, stroke, diabetes, cancer, and cognitive impairment). blncludes new onset of disability in self-care ADLs, limitations in mobility and social/recreational activities, and disability days in bed ( $\geq 7$ vs $0-6$ days), over 2.5 years. Comparisons were made between ACOS vs asthma alone and ACOS vs COPD alone, using multivariable logistic regression models that were adjusted for the same baseline covariates as described. Abbreviations: ACOS, asthma-COPD overlap syndrome; ADLs, activities of daily living; Cl, confidence interval.

long-standing or adult-onset asthma. ${ }^{1-3,6-17}$ This hypothesis would require spirometric confirmation and characterization of airway inflammation, which were not available in the MEPS cohort.

We additionally note that ACOS also had a higher frequency of cardiovascular disease and cardiac-based health care utilization (outpatient visits), but only when compared with asthma alone and not with COPD alone. The cardiovascular outcomes may relate to ACOS having a larger proportion of persons who were aged $\geq 65$ years and current smokers, as compared with asthma alone. Otherwise, both ACOS and COPD alone included a substantial proportion of older persons and exhibited similarly high rates of current smoking.

The MEPS dataset provides a unique opportunity to evaluate the population-based health burden of ACOS, relative to asthma alone and COPD alone. MEPS includes a contemporary and nationally representative sample of the US population, a large number of participants with asthma or
COPD, a wide age range, female and minority representation, and documentation of patient-reported outcomes and health care utilization. Moreover, because asthma and COPD were physician-diagnosed, rather than specifically established by spirometry, and because health care utilization was based on ICD-9 codes, the results of the current study have broad generalizability, including in primary care practice and older populations. Importantly, ACOS studies that are based on spirometry may have limited generalizability, given that primary care providers frequently lack access to spirometry as a means to establish obstructive airway disease, and given that older patients who are frail or cognitively impaired may have difficulty completing spirometry. ${ }^{33-36}$

Given the strengths of the MEPS dataset, we therefore posit that the current study informs public health policy and clinical practice regarding ACOS. At the population level, national surveys of obstructive airway disease in middle-aged and older persons should expand queries and analyses regarding the coexistence of risk factors for COPD and asthma, 

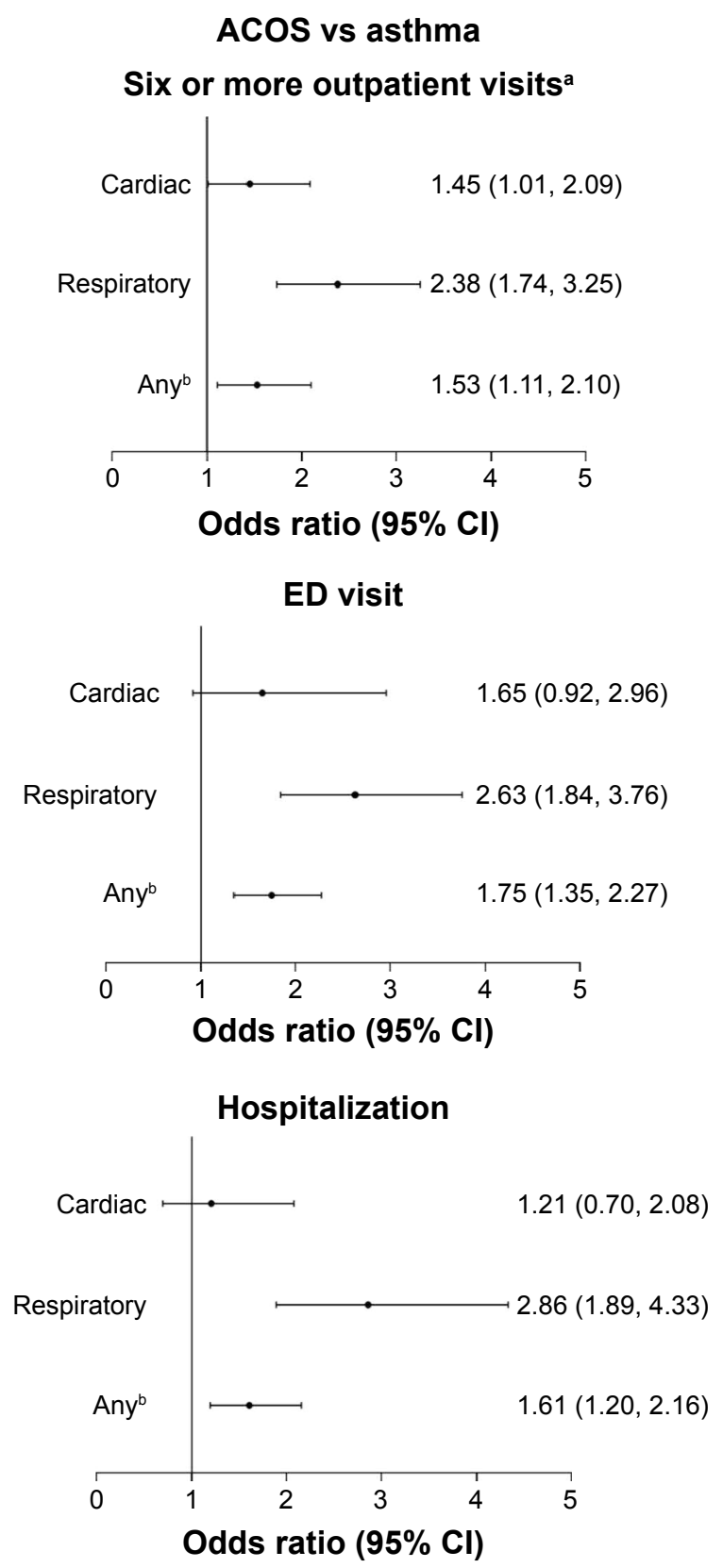

ACOS vs COPD

\section{Six or more outpatient visits ${ }^{a}$}
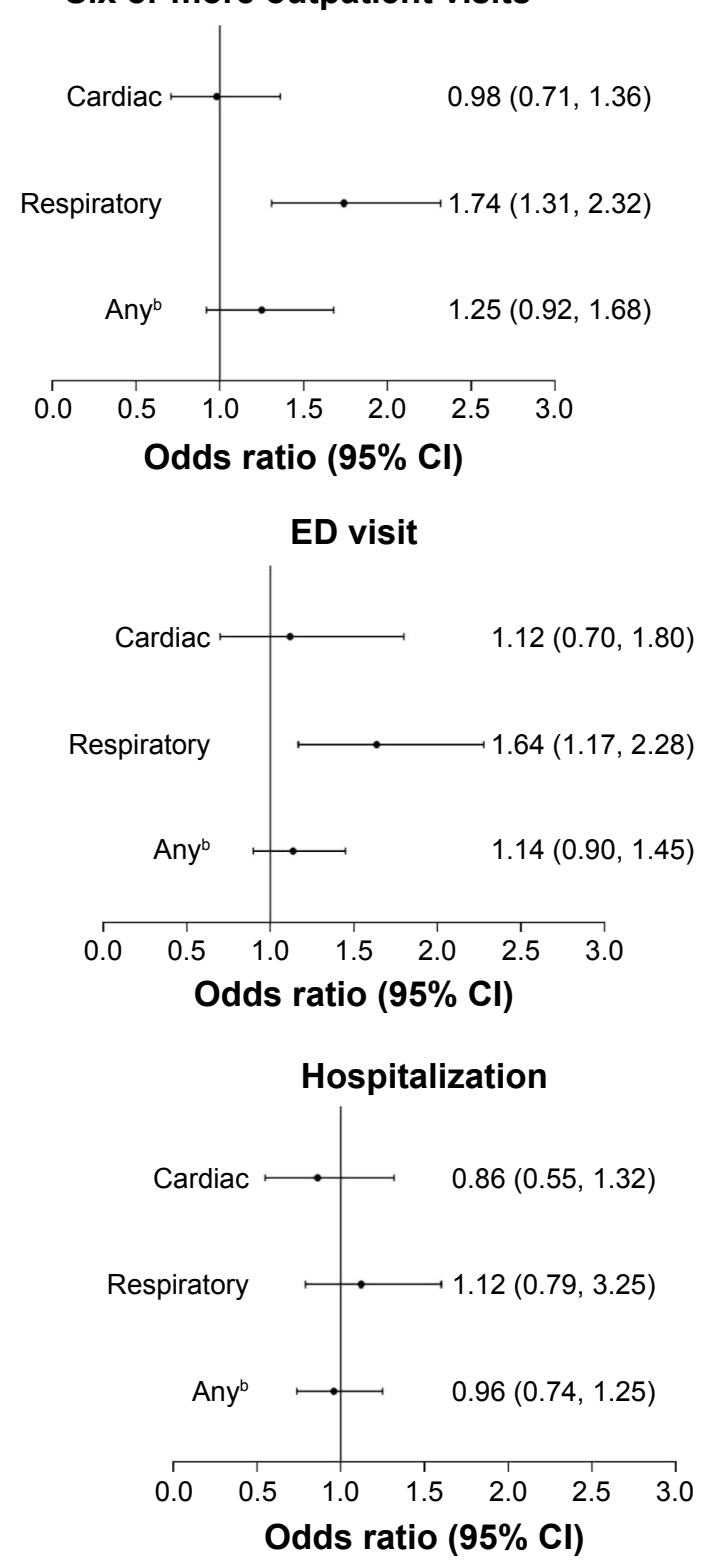

Figure 3 Comparisons of health care utilization over a follow-up period of 2.5 years: ACOS vs asthma alone and ACOS vs COPD alone.

Notes: Multivariable logistic regression models were adjusted for age, sex, race, ethnicity, marital status, education, income, medical insurance status, current smoking status, cardiovascular diseases, stroke, diabetes, cancer, and cognitive impairment. alncludes physician or hospital clinic visits, and corresponds to a frequency averaging more often than every 6 months. 'Due to any reason, including cardiac or respiratory.

Abbreviations: ACOS, asthma-COPD overlap syndrome; $\mathrm{Cl}$, confidence interval; ED, emergency department.

in order to better ascertain the epidemiology of ACOS. At the patient level, clinical practice guidelines that direct the management of obstructive airway disease in middle-aged and older persons should consider the impact of ACOS on patient-reported outcomes. ${ }^{26-28}$ We note that patient-reported outcomes are especially meaningful, as these often include functional activities that are highly valued by patients and are the basis for patient-centered care. ${ }^{26-28}$ We further note that patient-reported outcomes in older populations may be multifactorial and, in turn, are potentially modifiable by non-respiratory interventions such as addressing medicationrelated adverse effects and environmental barriers, and considering referrals to physical and occupational therapy, and social services. ${ }^{37}$

The current study also informs potential differences in the reported epidemiology of ACOS across countries. For example, using self-reported physician diagnoses that were similar to those in MEPS (US), a prior study ${ }^{19}$ of a 
Table 3 Health status scores for the PCS and MCS of the SF12v2, expressed as adjusted least squares means: asthma alone, COPD alone, and ACOS

\begin{tabular}{|c|c|c|c|c|}
\hline \multirow{3}{*}{$\begin{array}{l}\text { Obstructive } \\
\text { airway } \\
\text { disease }\end{array}$} & \multicolumn{4}{|c|}{ Health status (SF-I 2v2) } \\
\hline & \multicolumn{2}{|l|}{$\overline{\text { PCS }}$} & \multicolumn{2}{|l|}{ MCS } \\
\hline & $\begin{array}{l}\text { Adjusted least } \\
\text { squares mean } \\
\text { score }(95 \% \mathrm{Cl})^{\mathrm{a}}\end{array}$ & $P$-value ${ }^{\mathrm{b}}$ & $\begin{array}{l}\text { Adjusted least } \\
\text { squares mean } \\
\text { score }^{\mathrm{a}}\end{array}$ & $P$-value \\
\hline Asthma & $35.7(35.2,36.2)$ & $<0.000 \mathrm{I}^{\mathrm{b}}$ & $43.3(42.7,43.8)$ & $<0.000 I^{\prime}$ \\
\hline COPD & $28.9(28.1,29.7)$ & $0.0025^{c}$ & $4 I .3(40.4,42.1)$ & $0.1578^{c}$ \\
\hline ACOS & $26.9(25.8,28.0)$ & - & $40.3(39.2,41.5)$ & - \\
\hline
\end{tabular}

Notes: In normative data, the mean score is set to 50 ; thus, PCS and MCS scores $<50$

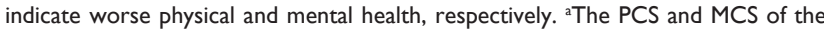
SF- I 2 2, which includes 12 items from the Medical Outcomes Study, were evaluated separately, with adjusted least squares mean scores calculated from longitudinal linear models, adjusted for time and the baseline covariates of age, sex, race, ethnicity, marital status, education, income, medical insurance status, current smoking status, cardiovascular diseases, stroke, diabetes, cancer, and cognitive impairment. ${ }^{b}$ Comparison is ACOS vs asthma alone. 'Comparison is ACOS vs COPD alone. Abbreviations: PCS, Physical Component Summary; MCS, Mental Component Summary; SF-12v2, Short Form 12, Version 2; ACOS, asthma-COPD overlap syndrome; $\mathrm{Cl}$, confidence interval.

nationally representative sample of the Chinese population reported that among participants who had asthma or COPD $(\mathrm{N}=2,793), 13.1 \%(366 / 2,793)$ had ACOS, whereas our MEPS analytical sample yielded an ACOS proportion of $17.4 \%(607 / 3,486)$. The same Chinese population study ${ }^{19}$ also reported that the health burden in ACOS was significantly greater than in COPD alone, but not in asthma alone, whereas our MEPS analytical sample showed that the health burden in ACOS was significantly greater relative to asthma alone and COPD alone.

The epidemiologic differences between the Chinese and American study samples suggest that the pathophysiology of ACOS may also differ. In particular, the Chinese study sample $^{19}$ included a substantial proportion of participants aged $<40$ years (our MEPS analytical sample did not, as COPD is rare in younger Americans) and, in addition, the Chinese study sample ${ }^{19}$ included a current smoking status that was consistently high across asthma alone, COPD alone, and ACOS $(40.7 \%, 32.5 \%$, and $37.7 \%$ vs our MEPS analytical sample of $14.0 \%, 39.3 \%$, and $38.1 \%$, respectively). We postulate that, in younger age groups, the pathophysiology of ACOS is likely to be more asthma predominant and less likely to include a component of severe COPD (lag time exists between smoking exposure and progression to severe COPD). Thus, if current smoking rates in China continue and are accompanied by increased aging of the population, the pathophysiology of ACOS may shift to a greater COPD component. Conversely, if the ongoing decline of smoking rates in the US continues and is accompanied by increased aging of the population, the pathophysiology of ACOS may shift to a greater asthma component (long-standing or adult-onset).

\section{Limitations}

Epidemiological surveys, such as MEPS and the earlier described US National Surveillance of Asthma and US Behavioral Risk Factor Surveillance System, have established asthma and COPD based on self-reported, physician diagnoses. ${ }^{4,5,29}$ As discussed earlier, this approach may be more generalizable to primary care and geriatric practice. In addition, within the context of a population-based study, our results suggest that self-reported, physician-diagnosed ACOS is clinically meaningful, given its association with respiratory-based health care utilization. Nonetheless, in an individual patient, the clinical assessment of obstructive airway disease requires objective confirmation by spirometric criteria, ${ }^{3,38}$ which was not available in MEPS.

Accordingly, we note that other studies have evaluated health burden in ACOS by using spirometric criteria from the Global initiative for Obstructive Lung Disease (GOLD), specifically to confirm COPD and to establish its severity. ${ }^{16-18,22-25}$ This approach, however, also has limitations. Although spirometry is an objective measure of lung function, it has limited generalizability in primary care practice and among older populations, as discussed earlier. ${ }^{33-36}$ Furthermore, even when spirometry is successfully completed, the GOLD spirometric criteria increasingly misidentify aging-related airflow limitation as COPD, starting at age $45-50$ years. ${ }^{39-43}$ In addition, there is no spirometric pattern of obstruction that can specifically distinguish COPD from asthma, including in the presence or absence of bronchodilator reversibility. ${ }^{1,44}$ Thus, there is a strong need to develop ACOS-based objective criteria, potentially including biomarkers that are generalizable to primary care practice and older populations; these may better establish the health burden at the population level, as well as better inform clinical management at the patient level..$^{45-48}$

We acknowledge other potential limitations to the MEPS dataset, including the omission of a smoking history based on pack-years and the omission of respiratory symptoms (especially dyspnea). In addition, MEPS first evaluated depressive symptoms and sensory impairments at round 2, rather than at baseline (round 1). These limitations have precluded a comparison of smoking exposure and respiratory symptoms between ACOS vs asthma alone and COPD alone, and have prevented the use of depressive symptoms and sensory impairments as covariates in multivariable models 
(ie, the measurement of these explanatory variables during the time of eligibility for the outcome did not allow for clear temporal precedence relative to the outcome).

\section{Conclusion}

Using baseline and longitudinal data from a large, nationally representative sample of the US population aged 40-85 years (MEPS), we have shown that ACOS is associated with increased health burden, including patient-reported outcomes and respiratory-based health care utilization, as compared with asthma alone and COPD alone. The increase in patientreported outcomes is especially meaningful, as these include functional activities that are highly valued by patients and are the basis for patient-centered care. ${ }^{26-28}$

\section{Acknowledgments}

The authors acknowledge the important contributions of the participants and staff of the MEPS, including the DHHS, AHRQ, and CDC. This work was supported by the US National Institute of Aging at the National Institutes of Health (R01 AG047891, R21 AG045148, P30 AG021342).

Dr Vaz Fragoso is a recipient of a Merit Award from the Department of Veterans Affairs.

\section{Disclosure}

The authors report no conflicts of interest in this work.

\section{References}

1. Postma DS, Rabe KF. The asthma-COPD overlap syndrome. $N$ Engl JMed. 2015;373(13):1241-1249.

2. Global Initiative for Asthma. Global strategy for asthma management and prevention (updated 2015). Available from: http://ginasthma.org/ archived-reports/. Accessed December 07, 2016.

3. Global Initiative for Chronic Obstructive Lung Disease. Global strategy for the diagnosis, management, and prevention of chronic obstructive pulmonary disease (updated 2015). Available from: http://goldcopd. org/archived-reports/. Accessed December 07, 2016.

4. Moorman JE, Akinbami LJ, Bailey CM, et al. National Surveillance of Asthma: United States, 2001-2010. Vital Health Stat 3. 2012;(35):1-58.

5. Kosacz NM, Punturieri A, Croxton TL, et al. Chronic obstructive pulmonary disease among adults - United States, 2011. MMWR. 2012;61(46): 938-943.

6. Pleasants RA, Ohar JA, Croft JB, et al. Chronic obstructive pulmonary disease and asthma-patient characteristics and health impairment. COPD. 2014;11(3):256-266.

7. Gibson PG, Simpson JL. The overlap syndrome of asthma and COPD: what are its features and how important is it? Thorax. 2009;64(8): 728-735.

8. de Marco R, Pesce G, Marcon A, et al. The coexistence of asthma and chronic obstructive pulmonary disease (COPD): prevalence and risk factors in young, middle aged and elderly people from the general population. PLoS One. 2013;8(5):e62985.

9. Papaiwannou A, Zarogoulidis P, Porpodis K, et al. Asthma-chronic obstructive pulmonary disease overlap syndrome (ACOS): current literature review. J Thorac Dis. 2014;6(S1):S146-S151.

10. Barnes PJ. Asthma-COPD overlap. Chest. 2016;149(1):7-8.
11. Gibson PG, McDonald VM. Asthma-COPD overlap 2015: now we are six. Thorax. 2015;70(7):683-691.

12. Hardin M, Cho M, McDonald ML, et al. The clinical and genetic features of COPD-asthma overlap syndrome. Eur Respir J. 2014; 44(2):341-350.

13. Hirota N, Martin JG. Mechanisms of airway remodeling. Chest. 2013; 144(3): 1026-1032.

14. de Nijs SB, Venekamp LN, Bel EH. Adult-onset asthma: is it really different? Eur Respir Rev. 2013;22(127):44-52.

15. Barrecheguren M, Esquinas C, Miravitlles M. The asthma-chronic obstructive pulmonary disease overlap syndrome (ACOS): opportunities and challenges. Curr Opin Pulm Med. 2015;21(1):74-79.

16. Hardin M, Silverman EK, Barr RG, et al; COPDGene Investigators. The clinical features of the overlap between COPD and asthma. Respir Res. 2011;12:127.

17. Cosio BG, Soriano JB, López-Campos JL, et al; CHAIN Study. Defining the asthma-COPD overlap syndrome in a COPD cohort. Chest. 2016; 149(1):45-52.

18. Nielsen M, Bårnes CB, Ulrik CS. Clinical characteristics of the asthmaCOPD overlap syndrome - a systematic review. Int J Chron Obstruct Pulmon Dis. 2015;10:1443-1454.

19. Ding B, DiBonaventura M, Karlsson N, Ling X. Asthma-chronic obstructive pulmonary disease overlap syndrome in the urban Chinese population: prevalence and disease burden using the 2010, 2012, and 2013 China National Health and Wellness Surveys. Int J Chron Obstruct Pulmon Dis. 2016;11:1139-1150.

20. Sorino C, Pedone C, Scichilone N. Fifteen-year mortality of patients with asthma-COPD overlap syndrome. Eur J Intern Med. 2016;34:72-77.

21. Kumbhare S, Pleasants R, Ohar JA, Strange C. Characteristics and prevalence of asthma/chronic obstructive pulmonary disease overlap in the United States. Ann Am Thorac Soc. 2016;13(6):803-810.

22. Tkacova R, Dai DLY, Vonk JM, et al. Airway hyperresponsiveness in chronic obstructive pulmonary disease: a marker of asthma-chronic obstructive pulmonary disease overlap syndrome? J Allergy Clin Immunol. Epub 2016 May 24.

23. Cosentino J, Zhao H, Hardin M, et al. Analysis of asthma-COPD overlap syndrome when defined on the basis of bronchodilator response and degree of emphysema. Ann Am Thorac Soc. 2016;13(9):1483-1489.

24. Lange P, Çolak Y, Ingebrigtsen TS, Vestbo J, Marott JL. Long-term prognosis of asthma, chronic obstructive pulmonary disease, and asthma-chronic obstructive pulmonary disease overlap in the Copenhagen City Heart study: a prospective population-based analysis. Lancet Respir Med. 2016;4(6):454-462.

25. Kauppi P, Kupiainen H, Lindqvist A, et al. Overlap syndrome of asthma and COPD predicts low quality of life. J Asthma. 2011;48(3): 279-285.

26. Gross NJ. Chronic obstructive pulmonary disease outcome measurements: what's important? What's useful? Proc Am Thorac Soc. 2005; 2(4):267-271.

27. Jones P, Miravitlles M, van der Molen T, Kulich K. Beyond FEV1 in COPD: a review of patient-reported outcomes and their measurement. Int J Chron Obstruct Pulmon Dis. 2012;7:697-709.

28. Haughney J, Partridge MR, Vogelmeier C, et al. Exacerbations of COPD: quantifying the patient's perspective using discrete choice modeling. Eur Respir J. 2005;26(4):623-629.

29. MEPS survey background. Rockville, MD: Agency for Healthcare Research and Quality. Available from: http://www.meps.ahrq.gov/ mepsweb/about_meps/survey_back.jsp. Accessed March 17, 2016.

30. Vaz Fragoso CA, McAvay G, Van Ness PH, et al. Phenotype of spirometric impairment in an aging population. Am J Respir Crit Care Med. 2016;193(7):727-735.

31. Kroenke K, Spitzer RL, Williams JBW. The patient health questionnaire-2: validity of a two-item depression screener. Med Care. 2003; 41(11):1284-1292.

32. Ware JE, Kosinski M, Keller SD. A 12-item short-form health survey: construction of scales and preliminary tests of reliability and validity. Med Care. 1996;34(3):220-233. 
33. Ferguson GT, Enright PL, Buist AS, Higgins MW. Office spirometry for lung health assessment in adults: a consensus statement from the National Lung Health Education Program. Chest. 2000;117(4):1146-1161.

34. Nishi SP, Wang Y, Kuo YF, Goodwin JS, Sharma G. Spirometry use among older adults with chronic obstructive pulmonary disease: 1999-2008. Ann Am Thorac Soc. 2013;10:565-573.

35. Joo MJ, Sharp LK, Au DH, Lee TA, Fitzgibbon ML. Use of spirometry in the diagnosis of COPD: a qualitative study in primary care. $C O P D$. 2013;10(4):444-449.

36. Allen SC, Yeung P. Inability to draw intersecting pentagons as a predictor of unsatisfactory spirometry technique in elderly hospital inpatients. Age Ageing. 2006;35(3):304-316.

37. Fried TR, Vaz Fragoso CA, Rabow MW. Caring for the older person with chronic obstructive pulmonary disease. JAMA. 2012;308(12): 1254-1263.

38. Vaz Fragoso CA, McAvay G, Gill TM, Concato J, Quanjer PH, Van Ness PH. Ethnic differences in respiratory impairment. Thorax. 2014;69(1):55-62.

39. Vaz Fragoso CA, Concato J, McAvay G, et al. The ratio of FEV1 to FVC as a basis for establishing chronic obstructive pulmonary disease. Am J Respir Crit Care Med. 2010;181(5):446-451.

40. Vaz Fragoso CA, Gill TM, McAvay G, Quanjer PH, Van Ness PH, Concato J. Respiratory impairment in older persons: when less means more. Am J Med. 2013;126(1):49-57.
41. Vaz Fragoso CA, McAvay G, Van Ness PH, et al. Phenotype of normal spirometry in an aging population. Am J Respir Crit Care Med. 2015; 192(7):817-825.

42. Stanojevic S, Wade A, Stocks J, et al. Reference ranges for spirometry across all ages: a new approach. Am J Respir Crit Care Med. 2008; 177(3):253-260.

43. Quanjer PH, Stanojevic S, Cole TJ, et al. Multi-ethnic reference values for spirometry for the 3-95 year age range: the global lung function 2012 equations. Eur Respir J. 2012;40(6):1324-1343.

44. Pellegrino R, Antonelli A, Mondino M. Bronchodilator testing: an endless story. Eur Respir J. 2010;35(5):952-954.

45. Ghebre MA, Bafadhel M, Desai D, et al. Biological clustering supports both "Dutch" and "British" hypotheses of asthma and chronic obstructive pulmonary disease. J Allergy Clin Immunol. 2015;135(1):63-72.

46. Bihlet AR, Karsdal MA, Bay-Jensen AC, et al. Clinical drug development using dynamic biomarkers to enable personalized health care in COPD. Chest. 2015;148(1):16-23.

47. Gauthier M, Ray A, Wenzel SE. Evolving concepts of asthma. Am J Respir Crit Care Med. 2015;192(6):660-668.

48. Soler-Cataluna JJ, Cosío B, Izquierdo JL, et al. Consensus document on the overlap phenotype COPD-asthma in COPD. Arch Bronconeumol. 2012;48(9):331-337.
International Journal of COPD

\section{Publish your work in this journal}

The International Journal of COPD is an international, peer-reviewed journal of therapeutics and pharmacology focusing on concise rapid reporting of clinical studies and reviews in COPD. Special focus is given to the pathophysiological processes underlying the disease, intervention programs, patient focused education, and self management protocols.

\section{Dovepress}

This journal is indexed on PubMed Central, MedLine and CAS. The manuscript management system is completely online and includes a very quick and fair peer-review system, which is all easy to use. Visit http://www.dovepress.com/testimonials.php to read real quotes from published authors. 